\title{
RESEARCH
}

Open Access

\section{Area deprivation and attachment to a general practitioner through centralized waiting lists: a cross-sectional study in Quebec, Canada}

Mélanie Ann Smithman ${ }^{1}$, Astrid Brousselle ${ }^{2}$, Nassera Touati ${ }^{3}$, Antoine Boivin ${ }^{4}$, Kareen Nour ${ }^{5}$, Carl-Ardy Dubois ${ }^{6}$, Christine Loignon ${ }^{1}$, Djamal Berbiche ${ }^{1}$ and Mylaine Breton ${ }^{1 *}$

\begin{abstract}
Background: Access to primary healthcare is an important social determinant of health and having a regular general practitioner (GP) has been shown to improve access. In Canada, socio-economically disadvantaged patients are more likely to be unattached (i.e. not have a regular GP). In the province of Quebec, where over $30 \%$ of the population is unattached, centralized waiting lists were implemented to help patients find a GP. Our objectives were to examine the association between social and material deprivation and 1) likelihood of attachment, and 2) wait time for attachment to a GP through centralized waiting lists.

Methods: A cross-sectional study was conducted in five local health networks in Quebec, Canada, using clinical administrative data of patients attached to a GP between June 2013 and May 2015 ( $n=24,958$ patients) and patients remaining on the waiting list as of May $2015(n=49,901)$, using clinical administrative data. Social and material area deprivation indexes were used as proxies for patients' socio-economic status. Multiple regressions were carried out to assess the association between deprivation indexes and 1) likelihood of attachment to a GP and 2) wait time for attachment. Analyses controlled for sex, age, local health network and variables related to health needs.

Results: Patients from materially medium, disadvantaged and very disadvantaged areas were underrepresented on the centralized waiting lists, while patients from socially disadvantaged and very disadvantaged areas were overrepresented. Patients from very materially advantaged and advantaged areas were less likely to be attached to a GP than patients from very disadvantaged areas. With the exception of patients from socially disadvantaged areas, all other categories of social deprivation were more likely to be attached to a GP compared to patients from very disadvantaged areas. We found a pro-rich gradient in wait time for attachment to a GP, with patients from more materially advantaged areas waiting less than those from disadvantaged areas.

Conclusion: Our findings suggest that there are socio-economic inequities in attachment to a GP through centralized waiting lists. Policy makers should take these findings into consideration to adjust centralized waiting list processes to avoid further exacerbation of health inequities.
\end{abstract}

Keywords: Physicians, family, Physicians, primary care, Primary health care, Health services accessibility, Socioeconomic factors, Healthy equity, Waiting lists, Cross-sectional, Canada, Quebec

\footnotetext{
* Correspondence: mylaine.breton@USherbrooke.ca

${ }^{1}$ Centre de recherche Charles-Le Moyne - Saguenay Lac-St-Jean sur les innovations en santé, Université de Sherbrooke, Longueuil Campus, 150 Place Charles-Le Moyne, Suite 200, Longueuil, Quebec J4K 0A8, Canada Full list of author information is available at the end of the article
}

(c) The Author(s). 2018 Open Access This article is distributed under the terms of the Creative Commons Attribution 4.0 International License (http://creativecommons.org/licenses/by/4.0/), which permits unrestricted use, distribution, and reproduction in any medium, provided you give appropriate credit to the original author(s) and the source, provide a link to the Creative Commons license, and indicate if changes were made. The Creative Commons Public Domain Dedication waiver (http://creativecommons.org/publicdomain/zero/1.0/) applies to the data made available in this article, unless otherwise stated. 


\section{Background}

Access to primary healthcare (PHC) is an important social determinant of health and improving access to $\mathrm{PHC}$ is considered to be a key strategy to reducing health inequities [1]. Although improving equity has been a central aim of health reforms in many OECD countries, inequities in access to PHC persist $[2,3]$. Even in countries with universal healthcare systems, like Canada, access has been found to vary, not only according to patients' medical need, but also according to factors such as income, education, social support and region of residence $[2,4,5]$. Having a regular general practitioner (GP) has been shown to improve access to PHC $[6,7]$.

However, many studies have found inequities in having a regular GP [6, 8-11] and in the likelihood of having visited a GP $[2,5,12]$, with patients with lower levels of income, education and social support having poorer access to a GP. Although in Canada GPs are key to accessing PHC as they are often the first point of contact to the healthcare system $[13,14], 15 \%$ of the population does not have a regular GP [14] - a large proportion compared to seven other OECD countries [15]. Quebec is the Canadian province with the highest proportion of patients reporting they do not have a regular GP [16], despite having a GP to population ratio higher than the Canadian average (116 vs. 111 GPs per 100,000 population) [17]. In Quebec, patients are formally attached to a GP, meaning that patients are officially registered on GPs' patient panels through an agreement signed on a voluntary basis during the first visit [17]. Formal attachment is strongly encouraged by the Ministry of Health and Social Services and is intended to increase GPs' accountability to their patients and to foster a continuous physician-patient relationship [18]. As of 2015, only $67.8 \%$ of the population was formally attached to a GP [19]. Over $65 \%$ of unattached patients report not being able to find a GP as the main reason they are unattached [10]. One of the main explanations for this large proportion of unattached patients is that GPs are required to dedicate a part of their time to regionally determined particular medical activities (activités médicales particulières) such as practicing in the emergency department or in long-term care facilities [17]. Consequently, GPs in Quebec have fewer hours available to care for attached patients [17].

In order to improve access to PHC, seven Canadian provinces (Ontario, Quebec, Manitoba, Nova Scotia, Prince-Edward-Island, British Columbia and New Brunswick) have implemented centralized waiting lists (CWLs) to help patients find a GP [20]. CWLs have a single intake point for patients' demand for a given service, may prioritize patients based on certain criteria, and help link patients to a provider from within a pool of providers. CWLs have been implemented in many fields of healthcare, namely for elective surgeries, transplants, occupational therapy, mental health services and referrals to specialists [21-25]. CWLs, with clear and respected guidelines for managing patient and provider lists, have been suggested as way to equalize wait times for patients with similar medical needs and to increase fairness in access to health services [26-28]. In other words, because CWLs standardize processes to access a given service, they could have the potential to reduce socio-economic inequities in attachment to GPs.

However, to our knowledge, there have been no studies on socio-economic inequities of CWLs for attachment to a GP. Yet, this information is essential to understand whether these CWLs are improving equity in access to a GP. In this paper, we aimed to fill this gap by analyzing the association between socio-economic factors and patient attachment to a GP through CWLs in Quebec. More specifically, the objectives were to examine the association between social and material deprivation and 1) likelihood of attachment, and 2) wait time for attachment to a GP through CWLs. First, we provide information on Quebec's CWLs and how they work; followed by contextual information on Quebec's healthcare system and a description of our methods; subsequently, we present descriptive statistics and findings for both objectives in the results section; we then discuss the main findings of our study with regards to inequities in registration on CWLs and attachment to a GP, the main implications for policy and the limitation of the study. This paper is part of a larger study evaluating the implementation and effects of CWLs for unattached patients in Quebec [29].

\section{Centralized waiting lists for attachment to a GP}

In Quebec, CWLs, the guichets d'accès pour la clientèle orpheline, were implemented in 2008 to increase the number of patients formally attached to GPs and prioritize attachment for patients with urgent health needs [30]. Over a million patients having been attached to a GP through these CWLs across the province [31]. Each CWL is staffed by a nurse, a clerk and a medical coordinator, a local GP, who manage the CWL for the local health network, according to general provincial guidelines.

CWLs are intended to help patients find a GP, as opposed to patients having to contact each clinic to see if a GP is available to attach them, but are not mandatory for GPs or patients. Unattached patients are not automatically registered on the CWLs. Those want to register on the CWLs can fill out an online or paper form or contact the CWL by phone. Health providers (e.g. physician at walk-in clinic or emergency department, social worker) may also fill out a CWL registration form on behalf of an unattached patient. Approximately 423,000 patients were waiting for attachment to a GP on a CWL 
in the province in 2015 , which represent about $17 \%$ of all unattached patients in Quebec. Other patients may not know about CWLs, may have chosen not to register on CWLs because they are looking for a GP on their own or because they are not looking for a GP. A patient may be formally attached to a GP through the CWLs or upon finding a GP that is willing to attach them through other channels such as at a walk-in clinic or through relatives or friends who ask their GP on the patient's behalf. GPs who are available to attach new patients may use the CWLs to find new patients or may choose to attach patients outside the CWLs (e.g. patients they meet at walk-in clinics or relatives of their current patients).

Upon registration, patients' contact, demographic and medical information is collected. Patients are asked to self-report medical conditions on the registration form (may also be filled out by a provider or over the phone as aforementioned). The CWL nurse uses this information, and may contact the patient by phone to obtain additional information, to assign patients one of five clinical priority levels, from priority 1 (most urgent) to 5 (least urgent), according to the guidelines in the provincial reference framework and clinical judgment. The clinical priority levels, based on patients' medical needs, were implemented to help prioritize attachment of patients with the most urgent needs and are linked to recommended wait times [30]. The wait times for each priority level are simply recommendations to help guide prioritization and are not enforced. Patient information is updated in the CWL database only if the patient contacts the CWL staff to notify the staff of any changes.

CWL patients are generally attached to a GP based on the availability of GPs in the area, clinical priority level, registration date and GPs' preferences and scope of practice. GPs' attachment of new patients through the CWLs is voluntary: GPs can contact the CWL intermittently to request the desired number of new patients and may specify their preferences for certain types of patients. The CWL nurses are responsible for selecting patients from the CWL for GPs who are willing to attach new patients and have some discretionary power in selecting patients for attachment, for instance to respect a GP's scope of practice or preference of certain types of patients. GPs may return patients they have not been able to reach, who have missed their first appointment or who do not fit their scope of practice to the CWL. A recent report in Quebec found that in about 35\% of cases where patients were sent back to the CWL without attachment to a GP it was because the GP felt they were medically or personally incompatible with the patient [32].

GPs receive a one-time financial incentive to attach patients from the CWL, with a larger incentive for patients with a least one of 19 medical vulnerability codes (e.g. cancer, mental health problems, intellectual disability) as defined by Quebec's Health Insurance Board, the Régie de l'Assurance Maladie du Québec (RAMQ), or being 70 years old or older [33]. Between 2013 and 2015, financial incentives for GPs for attaching patients from CWLs were: 100\$CAD for patients without a medical vulnerability code, $208 \$ C A D$ for patients with at least one medical vulnerability code or $250 \$ C A D$ for patients with a mental health or addiction problem [34]. GPs could receive financial incentives for attaching up to 150 new patients per year, with the exception of newly practicing GPs (4 years or less) who were not limited [34].

While CWLs for attachment to a GP have been implemented in most Canadian provinces, we have not found evidence of this type of CWL outside the Canadian context $[20,35]$. Within Canada, a recent study comparing the design of CWLs for attachment to a GP across seven provinces found that they had similar registration processes, but varied in how patients were prioritized for attachment and in financial incentives for GPs [20]. The study also reported that CWLs across Canada faced similar challenges in attaching vulnerable and complex patients and in the limited capacity to meet the demand for attachment [20]. Previous studies on Quebec's CWLs, using aggregated data have found that, despite larger financial incentives for attaching patients with health conditions, GPs showed a preference for attaching healthy patients [35] and that there are large variations in how CWLs perform across the province [30]. Moreover, although no research on CWL patients' socio-economic characteristics has been done to date, the equity of attachment to a GP through these CWLs has been called into question by the Auditor General in Quebec [36].

\section{Methods \\ Study setting \\ Context: Healthcare system in Quebec}

In Canada, each province and territory is responsible for organizing the delivery of most healthcare services [37]. Quebec, the second most populous Canadian province (population: 8.3 million) [38], provides publically funded universal health insurance for medical services. The Quebec Ministry of Health and Social Services determines the provincial healthcare system's priorities. The majority of GPs are paid on a fee-for-service basis. The large majority of primary care practices are managed by GPs who are self-employed, but paid by the government. Medical services provided by GPs are paid by the public provincial health directly. Approximately $20 \%$ of GPs are salaried and work in local community services centers which are managed by the government [35]. During the period under study, the province was divided into 94 geographically delimited local health networks which 
were created to address the different needs of the population in each area and implement a "population-based responsibility" in healthcare planning and delivery [39].

\section{Study design}

We conducted a cross-sectional study of CWLs for attachment of patients to a GP using clinical administrative data from Quebec, Canada.

\section{Data source}

We used administrative data from Quebec's information system for CWLs for unattached patients: the système d'information des guichets d'accès pour la clientele orpheline (SIGACO) database. This database compiles information for every patient requesting a GP through the CWLs. The data available in the SIGACO database is limited to attachment to GPs through CWLs and does not contain information on attachment outside CWLs.

Data is entered manually in the SIGACO system by the CWL's secretary or nurse in each local health network. We extracted individual patient data from five CWLs, which were selected to compare and contrast the implementation and performance of CWLs with varying results for indicators such as number of patients attached to a GP, number of patients on the waiting list and proportion of medically vulnerable patients attached to a GP [30], as part of the larger study on CWLs [29]. Four of the five CWLs were located in urban local health networks (A, B, C and E) and one in a semi-urban (D) local health network. In total, these local health networks represent about $12 \%$ of Quebec's population.

Data was extracted for two groups of patients: those attached to a GP through one of the five CWLs between June 1st, 2013 and May 31st, $2015(n=24,958)$, and those who remained on one of the five CWLs (i.e. still waiting for attachment) as of May 31st, 2015 ( $n=49$, 901). This period was selected because it represented the most recent data available at the time of the study and because changes in financial incentives and CWL guidelines had major impact on pre-2013 attachment [35].

\section{Variables}

\section{Dependent variables}

Our first dependent variable was likelihood of attachment to a GP through CWLs versus remaining on the waiting list, categorized as follows: 1 ) attached to a GP $(n=24,958)$ and 2) waiting for attachment $(n=49,901)$. Our second dependent variable was wait time for attachment to a GP through CWLs. Only patients attached to a GP during the period under study (June 2013 to May 2015) were included in this analysis $(n=24,958)$, because their total wait time could be calculated, whereas total wait time for attachment for patients remaining on the waiting list was unknown. Wait time was defined as the number of days between the date of registration on the CWL, which could precede the study period, and date of attachment to a GP.

\section{Main independent variables: Social and material deprivation}

Our two main independent variables are social and material deprivation. Health services administrative databases created by provincial authorities generally do not contain socio-economic data on individual patients. To make up for this shortcoming, geographic proxies, socio-economic data from a census or large health survey linked to small geographic areas, are commonly used to study inequities [11, 40-49]. In Quebec, a social and material deprivation index was developed in the late 1990s by Pampalon and collaborators to overcome the absence of socio-economic information in administrative databases and was intended for use in research and health planning $[11,41,49,50]$. Deprivation is conceptualized as "a state of observable and demonstrable disadvantage relative to local community or the wider society or nation to which the individual, family or group belongs" $[49,51]$. The deprivation index is based on data from the Canadian census and relates to dissemination areas with populations of 400 to 700 individuals [49]. It includes socio-economic indicators grouped along two dimensions - material and social. Material deprivation is based on average personal income, proportion of persons without a high school diploma and employment ratio. Social deprivation is calculated using the proportion of persons living alone, the proportion of individuals who are divorced, widowed or separated and the proportion of single-parent families. Although the area-based deprivation index has been shown to underestimate inequities when compared to individual-level data, it produces statistically reliable estimates consistent with individual indicators and detects sizeable inequities between groups [52]. This index has been widely used to examine inequities in health and healthcare in Quebec and other Canadian provinces [41, 53-57]. For our analyses, we used the 2006 version of the deprivation index and linked it to patients' postal codes in the SIGACO database. While a 2011 version of the index exists, it was calculated using the voluntary Canadian National Household Survey that replaced the census in 2011 and had a very high non-response rate which may have led to non-response bias and inaccurate results [58].

\section{Control variables}

We included sex and age as demographic variables. Based on previous work showing large variations in performance of CWLs between local health networks and no association between CWLs' performance and proportion of the population attached to a GP [30], we controlled for local health network (A to E) but not for 
proportion of population attached to a GP per local health network. To control for health needs, we included: medical vulnerability (i.e. the presence or absence of at least one of 19 medical vulnerability codes or being older of 70 year old); clinical priority level (Priority 1 - most urgent, to Priority 5 - least urgent); and the total number of medical vulnerability codes as a proxy for complexity. In addition, we included the presence/ absence of mental health problems (excluding major depression), major depression, addiction, intellectual disability, diabetes, COPD and active cancer. These conditions were identified as particularly important to control for because of their influence on likelihood and wait time for attachment in preliminary analyses and/or because they were identified as particularly influential in interviews with key stakeholders (GPs, CWL staff, policy makers) (unpublished results). For instance, stakeholders said that patients with active cancer were generally very rapidly attached, while patients with mental health or addictions problems waited much longer or remained on the waiting list because it was difficult to find GPs who would attach them (unpublished results). For the likelihood of attachment, we also controlled for wait time on the CWL: the number of days between registration on the CWL and May 31st, 2015 for patients who remained on the CWL and the number of days between registration and attachment for patients who had been attached to a GP. For the analysis on wait time for attachment, we were able to identify GPs to whom patients had been attached by their unique identifier in the database ( $n=469$ physicians) and, therefore, to account for their potential influence in the statistical model (see below).

\section{Analysis}

We found less than 5\% missing data and the listwise deletion method was employed for incomplete records. Univariate analyses (percentages, means, and standard errors) were used to describe the population under study. In order to assess the association between each independent and dependent variables, bivariate analyses were conducted (chi-square, t-test). To examine the association between social and material deprivation and the likelihood of attachment to a GP through CWLs, we used a multiple logistic regression, controlling for demographic, local health network, medical need and wait time variables, using forced entry, given that our selection of variables had a strong theoretical reasoning based on previous work (see Eq. 1). Odds ratios with 95\% confidence intervals were determined for all variables.

Equation 1 Multiple logistic regression of likelihood of attachment to a GP through CWLs

$$
\begin{aligned}
& \log \left[\frac{\mathrm{P}}{1-\mathrm{P}}\right]=\beta_{0}+\beta_{1}{ }^{*} X_{\text {social deprivation }}+\beta_{2}{ }^{*} X_{\text {material deprivation }}+\beta_{3}{ }^{*} X_{\text {sex }}+\beta_{4} \\
& { }^{*} X_{\text {age }}+\beta_{5}{ }^{*} X_{\text {local health network }}+\beta_{6}{ }^{*} X_{\text {medical vulnerability }}+\beta_{7} \\
& { }^{*} X_{\text {clinical priority }}+\beta_{8}{ }^{*} X_{\text {number of health conditions }}+\beta_{9}{ }^{*} X_{\text {health conditions }}+\beta_{10} \\
& { }^{*} X_{\text {wait time }}+\varepsilon
\end{aligned}
$$

P: Probability of being attached; $\beta_{\mathrm{k}}$ : beta coefficient for each variable; $\mathrm{X}_{\mathrm{k}}$ : variables included in the logistic regression model; $\varepsilon$ : error.

For wait time for attachment to a GP through CWLs, the intraclass correlation related to GPs to whom patients had been attached was of $18 \%$, indicating that a substantial proportion of variance in wait times was due to the GP. Because the intraclass correlation was larger than 5\% [59], we used a multilevel model for examine the association between deprivation and wait time for attachment to a GP. We included GPs' unique identifier at the higher level of our model, but no other variables related to GPs were included. We used a generalized linear mixed model, controlling for demographic, local health network and medical need variables (see Eq. 2).

Equation 2 Multilevel linear regression of wait time for attachment to a GP through CWLs

$$
\begin{aligned}
& \mathrm{Y}=\beta_{00}+\beta_{10}{ }^{*} X_{\text {social deprivation }}+\beta_{20}{ }^{*} X_{\text {material deprivation }}+\beta_{30}{ }^{*} X_{\text {sex }}+\beta_{40}{ }^{*} X_{\text {age }} \\
& +\beta_{50}{ }^{*} X_{\text {local health network }}+\beta_{60}{ }^{*} X_{\text {medical vulnerability }}+\beta_{70}{ }^{*} X_{\text {clinical priority }} \\
& +\beta_{80}{ }^{*} X_{\text {number of health conditions }}+\beta_{90}{ }^{*} X_{\text {health conditions }}+\mu_{0 j}+\varepsilon_{i j}
\end{aligned}
$$

Y: Wait time for attachment; $\beta_{\mathrm{k}}$ : beta coefficient for each variable; $X_{\mathrm{k}}$ : variables included in the linear mixed model; $\varepsilon$ : error.

For both analyses, we conducted sensitivity analysis with interaction terms between social and material deprivation, and between both deprivation indexes and all the control variables. The interaction terms were non-significant and had little influence of the estimates in the statistical models and were, therefore, excluded from the final analyses. All variance inflation factors (VIF) were inferior to 5 suggesting that there was no issues with multicollinearity in the logistic regression model. We used SAS 9.3 for all statistical analyses.

\section{Results}

A total of 24, 958 patients were attached to 469 GPs between June 1st, 2013 and May 31st, 2015, while 49, 901 patients remained on the five CWLs as of May 31st, 2015. Average wait time (standard deviation-s.d.) for patients attached to a GP was 284 days (s.d. 350) and 458 days (s.d. 359) for patients waiting for attachment $(p<0.0001)$.

Table 1 compares patient characteristics in the two groups. Patients waiting for attachment differed significantly from patients attached to a GP. With regards to 
material and social deprivation, per definition, each deprivation quintile represents $20 \%$ of Quebec's population. Compared to the distribution of deprivation indexes at the provincial level, we found that patients from the most materially advantaged areas (richest) represented $30.6 \%$ of patients on the waiting lists while patients from the most materially disadvantaged areas (poorest) represented a mere $11.65 \%$, with a pro-rich gradient in proportions of patients on the waiting lists. This difference seems to be lessened among patients attached to a GP $(23.27 \%$ most advantaged vs. $18.88 \%$ most disadvantaged). Conversely, we found that patients from very socially advantaged areas were slightly underrepresented $(18.82 \%)$ and patients from very socially disadvantaged areas were overrepresented on the waiting list $(29.49 \%)$ and with an even larger difference among patients attached to a GP $(12.13 \%$ very advantaged vs. $37.78 \%$ very disadvantaged).

A larger proportion of patients waiting for attachment were female (54\%). Patients waiting for attachment were slightly older $(1.27$ years, $p<0.0001)$. The distribution of the two groups across local health networks was also significantly different. For instance, patients from LHN A made up $23.03 \%$ of patients waiting for attachment and only $8.23 \%$ of patients attached to a GP, while those from LHN E represented $8.67 \%$ of patients waiting for attachment and $32.42 \%$ of those attached to a GP. A much larger proportion of patients attached to a GP were medically vulnerable, had been identified as clinical priority 1,2 or 3 and had one or more health conditions. The prevalence of mental health problems, major depression, addiction, intellectual disability, active cancer, diabetes and COPD was higher in patients attached to a GP.

Table 2 shows the distribution of patients across material and social deprivation quintiles, with regards to the other variables under study. A larger proportion of CWL patients from both materially and socially disadvantaged areas had been attached to a GP, compared to more advantaged areas. We observed a small gradient in mean age, with patients from socially and materially very disadvantaged areas being slightly younger. Patients from each deprivation quintile were unequally distributed across Local Health Networks, namely with Local Health Network E accounting for a large proportion of both socially and materially very disadvantaged patients. A smaller proportion of very socially and materially disadvantaged patients were prioritized as least urgent. A small gradient in the mean number of health conditions was observed, with patients from socially and materially disadvantaged areas having more health conditions. A slightly larger proportion of patients from both socially and materially very disadvantaged areas had mental health and addiction problems. In addition, a larger proportion of patients from materially very disadvantaged areas had an intellectual disability, diabetes and COPD.

\section{Likelihood of attachment to a GP}

Results of the analysis for our first objective (i.e. to examine the association between deprivation and the likelihood of attachment to a GP) are presented in Table 3. Likelihood of attachment to a GP was significantly lower for materially very advantaged (OR $0.75, \mathrm{CI}$ $0.70-0.81$ ) and advantaged patients (OR 0.88, CI 0.82$0.95)$ compared to very disadvantaged patients. In contrast, for social deprivation, likelihood of attachment was higher for very advantaged (OR 1.23, CI 1.15-1.32), advantaged (OR 1.21, CI 1.13-1.30) and medium (OR 1.19 , CI 1.11-1.27) compared to very disadvantaged.

While our focus is on material and social deprivation, several results relating to our control variables are of interest. Males were slightly more likely to be attached and increase in age slightly decreased likelihood of attachment. Moreover, likelihood of attachment to a GP varied between local health networks.

Medically vulnerable patients were 2.56 times more likely to be attached to a GP (OR 2.56, CI 2.26-2.84). Likelihood of attachment increased with urgency of clinical priority, with Priority 1 (most urgent) patients being nearly 86 times more likely to be attached to a GP than Priority 5 patients. Patients with active cancer (OR 1.54, CI 1.36-1.74) and with diabetes (OR 1.46, CI 1.32-1.62) were more likely to be attached to a GP, while patients with mental health problems (OR 0.42, CI 0.38-0.46), major depression (OR 0.85, CI 0.73-0.98), addiction (OR 0.39, CI 0.31-0.49) or intellectual disability (OR 0.72 , CI $0.54-0.96$ ) were more likely to remain on the waiting list.

\section{Wait time for attachment to a GP}

Table 4 presents the results for our second objective of assessing the association between deprivation and wait time for attachment to a GP. The multilevel linear regression model included fixed patients level variables (level-1). While no GP level variables (level 2) were included in the model, the intra-class correlation suggests that $18 \%$ of the variance in wait times is explained by the GP to whom patients were attached. With regards to material deprivation, we observed a gradient in coefficients. For example, patients from very advantaged areas waiting 34 days less than patients from very disadvantaged areas $(p<0.001)$. Wait times for patients from medium socially disadvantaged areas waited about 15 days less than those for very socially disadvantaged areas $(p=0.0061)$. Wait times were not significantly different between patients from very socially disadvantaged areas and patients from other quintiles of deprivation. 
Table 1 Descriptive statistics for population under study (patients attached to a GP through centralized waiting list $(n=24,958)$; patients waiting on centralized waiting lists ( $n=49$, 901), June 2013 to May 2015)

\begin{tabular}{|c|c|c|c|c|c|}
\hline Descriptive statistics & & $\begin{array}{l}\text { All CWL patients (waiting for } \\
\text { attachment \& attached) (\%) }\end{array}$ & $\begin{array}{l}\text { Patients waiting for } \\
\text { attachment (\%) }\end{array}$ & $\begin{array}{l}\text { Patients attached } \\
\text { to a GP }(\%)\end{array}$ & $\mathrm{P}$ (Chi-2 or t-test) \\
\hline \multirow[t]{5}{*}{ Material deprivation $^{\mathrm{a}}$} & Very advantaged & 28.16 & 30.60 & 23.27 & $<0.0001$ \\
\hline & Advantaged & 21.96 & 23.16 & 19.56 & \\
\hline & Medium & 17.37 & 17.05 & 18.01 & \\
\hline & Disadvantaged & 18.46 & 17.55 & 20.28 & \\
\hline & Very disadvantaged & 14.06 & 11.65 & 18.88 & \\
\hline \multirow[t]{5}{*}{ Social deprivation ${ }^{\mathrm{b}}$} & Very advantaged & 16.59 & 18.82 & 12.13 & $<0.0001$ \\
\hline & Advantaged & 13.12 & 14.03 & 11.30 & \\
\hline & Medium & 15.28 & 16.08 & 13.67 & \\
\hline & Disadvantaged & 22.76 & 21.57 & 25.12 & \\
\hline & Very disadvantaged & 32.25 & 29.49 & 37.78 & \\
\hline $\operatorname{Sex}(\%)$ & Female & 53.58 & 54.07 & 52.60 & 0.0001 \\
\hline Mean age $(\text { s.d. })^{c}$ & & $42.13(22.11)$ & $42.56(19.03)$ & $41.29(27.24)$ & $<0.0001$ \\
\hline \multirow[t]{5}{*}{ Local health network (\%) } & A & 18.01 & 23.03 & 8.23 & $<0.0001$ \\
\hline & B & 12.37 & 10.58 & 15.95 & \\
\hline & C & 24.30 & 31.16 & 10.59 & \\
\hline & $\mathrm{D}$ & 28.64 & 26.56 & 32.80 & \\
\hline & $\mathrm{E}$ & 16.59 & 8.67 & 32.42 & \\
\hline \multirow[t]{2}{*}{ Medical vulnerability (\%) } & Vulnerable & 31.38 & 23.95 & 46.23 & $<0.0001$ \\
\hline & Non-vulnerable & 68.62 & 76.05 & 53.77 & \\
\hline \multirow[t]{5}{*}{ Clinical priority (\%) } & 1 (most urgent) & 1.95 & 0.37 & 4.62 & $<0.0001$ \\
\hline & 2 & 15.86 & 6.33 & 31.90 & \\
\hline & 3 & 12.57 & 8.63 & 19.22 & \\
\hline & 4 & 25.54 & 27.06 & 22.98 & \\
\hline & 5 (least urgent) & 44.08 & 57.61 & 21.29 & \\
\hline \multirow[t]{3}{*}{ Number of health conditions $(\%)^{f}$} & None & 72.48 & 79.20 & 59.04 & $<0.0001$ \\
\hline & 1 condition & 18.28 & 13.56 & 27.71 & \\
\hline & $\geq 2$ conditions & 9.24 & 7.24 & 13.25 & \\
\hline \multirow[t]{7}{*}{ Health conditions (\%) } & Mental health ${ }^{g}$ & 6.23 & 5.39 & 7.89 & $<0.0001$ \\
\hline & Major depression & 1.91 & 1.47 & 2.80 & $<0.0001$ \\
\hline & Addiction & 0.81 & 0.75 & 0.91 & 0.02 \\
\hline & Active cancer & 3.90 & 2.29 & 7.11 & $<0.0001$ \\
\hline & Intellectual disability & 0.48 & 0.40 & 0.62 & $<0.0001$ \\
\hline & Diabetes & 5.35 & 3.52 & 9.02 & $<0.0001$ \\
\hline & COPD & 3.86 & 3.19 & 5.22 & $<0.0001$ \\
\hline Mean wait time (s.d.) & & - & $458(359)$ & $284(350)$ & $<0.0001$ \\
\hline
\end{tabular}

${ }^{a}$ Area-based material deprivation

${ }^{\mathrm{b}}$ Area-based social deprivation

cs.d.: standard deviation

${ }^{\mathrm{d}}$ Medical vulnerability is determined by the presence of at least one of 19 medical vulnerability codes, as pre-determined by the RAMQ, or being older of 70 year old

${ }^{\text {e}}$ Clinical priority level as assigned by the CWLs' nurses (ranging from Priority 1 - most urgent, to Priority 5 - least urgent)

${ }^{f}$ Number of medical vulnerability codes present

${ }^{9}$ Mental health problems excluding major depression

${ }^{h}$ Mean wait time in days: For patients waiting for attachment - number of days between CWL registration and May 31st, 2015; For patients attached to a GP -

number of days between CWL registration and attachment. Not provided for all patients because measured differently for each group 


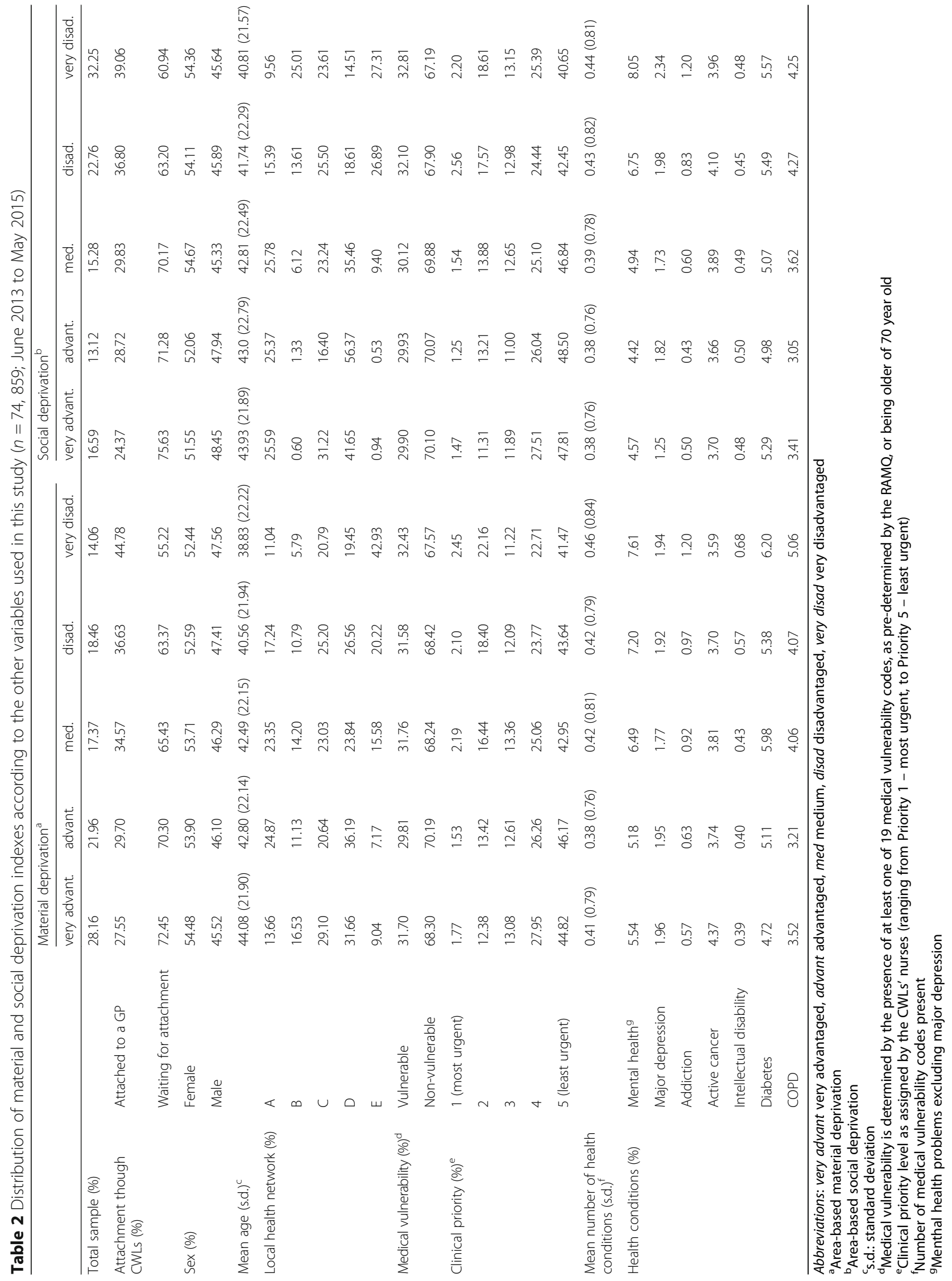


Table 3 Factors associated with likelihood of attachment to a GP through centralized waiting list among all subjects ( $\mathrm{n}=74$, 859; June 2013 to May 2015) (multiple logistic regression)

\begin{tabular}{|c|c|c|c|c|}
\hline \multirow[t]{2}{*}{ Variables in the model } & & \multicolumn{3}{|c|}{ Attachment to a GP } \\
\hline & & Adjusted ORi & $95 \% \mathrm{Cl}$ & $P$-value \\
\hline \multirow[t]{5}{*}{ Material deprivation $^{a}$} & Very advantaged & 0.75 & $0.70 ; 0.81$ & $<.0001$ \\
\hline & Advantaged & 0.88 & $0.82 ; 0.95$ & 0.0004 \\
\hline & Medium & 0.99 & $0.92 ; 1.06$ & 0.77 \\
\hline & Disadvantaged & 0.99 & $0.91 ; 1.06$ & 0.67 \\
\hline & Very disadvantaged (ref.) ${ }^{b}$ & & & \\
\hline \multirow[t]{5}{*}{ Social deprivation ${ }^{c}$} & Very advantaged & 1.23 & $1.15 ; 1.32$ & $<.0001$ \\
\hline & Advantaged & 1.21 & $1.13 ; 1.30$ & $<.0001$ \\
\hline & Medium & 1.19 & $1.11 ; 1.27$ & $<.0001$ \\
\hline & Disadvantaged & 1.04 & $0.99 ; 1.10$ & 0.11 \\
\hline & Very disadvantaged (ref.) ${ }^{b}$ & & & \\
\hline \multirow[t]{2}{*}{ Sex } & Male & 1.07 & $1.02 ; 1.11$ & 0.0019 \\
\hline & Female (ref.) $)^{\mathrm{b}}$ & & & \\
\hline Age & & 0.99 & $0.98 ; 0.99$ & $<.0001$ \\
\hline \multirow[t]{5}{*}{ Local health network } & A & 0.12 & $0.11 ; 0.13$ & $<.0001$ \\
\hline & B & 0.42 & $0.39 ; 0.45$ & $<.0001$ \\
\hline & C & 0.04 & $0.04 ; 0.05$ & $<.0001$ \\
\hline & $\mathrm{D}$ & 0.43 & $0.41 ; 0.46$ & $<.0001$ \\
\hline & $E(r e f .)^{b}$ & & & \\
\hline \multirow[t]{2}{*}{ Medical vulnerability ${ }^{d}$} & Vulnerable & 2.56 & $2.26 ; 2.84$ & $<.0001$ \\
\hline & Non-vulnerable (ref.) ${ }^{b}$ & & & \\
\hline \multirow[t]{5}{*}{ Clinical priority } & 1 (most urgent) & 85.88 & $70.32 ; 104.89$ & $<.0001$ \\
\hline & 2 & 13.43 & $12.39 ; 14.56$ & $<.0001$ \\
\hline & 3 & 6.82 & $6.22 ; 7.48$ & $<.0001$ \\
\hline & 4 & 2.13 & $2.03 ; 2.25$ & $<.0001$ \\
\hline & 5 (least urgent) (ref.) ${ }^{b}$ & & & \\
\hline Number of health conditions ${ }^{f}$ & & 0.95 & $0.91 ; 1.00$ & 0.07 \\
\hline \multirow[t]{8}{*}{ Health conditions } & Mental health ${ }^{9}$ & 0.42 & $0.38 ; 0.46$ & $<.0001$ \\
\hline & Major depression & 0.85 & $0.73 ; 0.98$ & 0.03 \\
\hline & Addiction & 0.39 & $0.31 ; 0.49$ & $<.0001$ \\
\hline & Active cancer & 1.54 & $1.36 ; 1.74$ & $<.0001$ \\
\hline & Intellectual disability & 0.72 & $0.54 ; 0.96$ & 0.03 \\
\hline & Diabetes & 1.46 & $1.32 ; 1.62$ & $<.0001$ \\
\hline & COPD & 1.00 & $0.89 ; 1.12$ & 0.98 \\
\hline & Absence of condition (ref.) $)^{b}$ & & & \\
\hline Wait time (days) ${ }^{h}$ & & 1.00 & $0.99 ; 1.00$ & 0.54 \\
\hline
\end{tabular}

${ }^{a}$ Area-based material deprivation

bef:: reference category

'Area-based social deprivation

${ }^{\mathrm{d}}$ Medical vulnerability is determined by the presence of at least one of 19 medical vulnerability codes, as pre-determined by the RAMQ, or being older of 70 year old

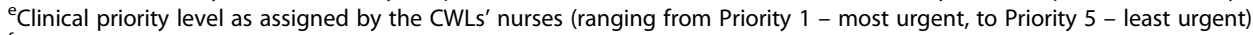

${ }^{f}$ Number of medical vulnerability codes present

${ }^{9}$ Mental health problems excluding major depression

hWait time: For patients waiting for attachment - number of days between CWL registration and May $31^{\text {st }}, 2015$; For patients attached to a GP - number of days between CWL registration and attachment

'Adjusted OR: Odds ratio adjusted for all other variables presented in the table 
Table 4 Factors associated with the wait time (days) for attachment to a GP among subjects who have been attached to a GP through centralized waiting list ( $n=24,958$; June 2013 to May 2015) (Multilevel linear regression)

\begin{tabular}{|c|c|c|c|c|}
\hline \multirow[t]{2}{*}{ Variables in the model } & & \multicolumn{3}{|c|}{ Wait time for attachment to a GP } \\
\hline & & Coefficient Beta $^{\text {h }}$ & $95 \% \mathrm{Cl}$ & P-value \\
\hline \multirow[t]{5}{*}{ Material deprivation $^{a}$} & Very advantaged & -34.20 & $-45.82 ;-22.59$ & $<.0001$ \\
\hline & Advantaged & -24.92 & $-36.74 ;-13.10$ & $<.0001$ \\
\hline & Medium & -19.10 & $-30.67 ;-7.54$ & 0.0012 \\
\hline & Disadvantaged & -13.59 & $-24.70 ;-2.48$ & 0.0166 \\
\hline & Very disadvantaged (ref.) ${ }^{b}$ & & & \\
\hline \multirow[t]{5}{*}{ Social deprivation ${ }^{c}$} & Very advantaged & -7.14 & $-19.98 ; 5.69$ & 0.2750 \\
\hline & Advantaged & -7.75 & $-20.82 ; 5.33$ & 0.2455 \\
\hline & Medium & -15.92 & $-27.29 ;-4.55$ & 0.0061 \\
\hline & Disadvantaged & -5.14 & $-13.91 ; 3.63$ & 0.2505 \\
\hline & Very disadvantaged (ref.) ${ }^{b}$ & & & \\
\hline \multirow[t]{2}{*}{ Sex } & Female & -4.34 & $-11.14 ; 2.45$ & 0.2098 \\
\hline & Male $(\text { ref. })^{b}$ & & & \\
\hline Age & & 1.30 & $1.10 ; 1.50$ & $<.0001$ \\
\hline \multirow[t]{5}{*}{ Local health network } & A & 266.20 & $157.52 ; 374.89$ & 0.0089 \\
\hline & B & 7.68 & $-85.38 ; 100.73$ & 0.7566 \\
\hline & C & 182.57 & $88.95 ; 276.19$ & 0.0139 \\
\hline & $\mathrm{D}$ & -41.65 & $-121.13 ; 37.83$ & 0.1528 \\
\hline & $E(r e f .)^{b}$ & & & \\
\hline \multirow[t]{2}{*}{ Medical vulnerability ${ }^{\mathrm{d}}$} & Vulnerable & -73.72 & $-59.46 ;-87.99$ & $<.0001$ \\
\hline & Non-vulnerable (ref.) ${ }^{b}$ & & & \\
\hline \multirow[t]{5}{*}{ Clinical priority $^{e}$} & 1 (most urgent) & -441.72 & $-464.72 ;-418.72$ & $<.0001$ \\
\hline & 2 & -296.38 & $-308.66 ;-284.10$ & $<.0001$ \\
\hline & 3 & -246.75 & $-261.71 ;-231.79$ & $<.0001$ \\
\hline & 4 & -107.92 & $-118.98 ;-96.85$ & $<.0001$ \\
\hline & 5 (least urgent) (ref.) $)^{b}$ & & & \\
\hline Number of health conditions ${ }^{f}$ & & -12.3450 & $-19.77 ;-4.92$ & 0.0011 \\
\hline \multirow[t]{8}{*}{ Health conditions } & Mental health ${ }^{g}$ & 78.50 & $63.00 ; 94.01$ & $<.0001$ \\
\hline & Major depression & 21.01 & $-0.83 ; 42.84$ & 0.06 \\
\hline & Addiction & 122.07 & $84.46 ; 159.69$ & $<.0001$ \\
\hline & Active cancer & 1.28 & $-14.79 ; 17.36$ & 0.8754 \\
\hline & Intellectual disability & 70.85 & $26.88 ; 114.82$ & 0.0019 \\
\hline & Diabetes & -5.47 & $-19.98 ; 9.05$ & 0.4591 \\
\hline & COPD & 12.38 & $-4.94 ; 29.69$ & 0.1604 \\
\hline & Absence of condition (ref.) $)^{b}$ & & & \\
\hline
\end{tabular}

${ }^{a}$ Area-based material deprivation

${ }^{\text {b}}$ Ref.: reference category

${ }^{c}$ Area-based social deprivation

${ }^{d}$ Medical vulnerability is determined by the presence of at least one of 19 medical vulnerability codes, as pre-determined by the RAMQ, or being older of

70 year old

${ }^{\text {e}}$ Clinical priority level as assigned by the CWLs' nurses (ranging from Priority 1 - most urgent, to Priority 5 - least urgent)

${ }^{f}$ Number of medical vulnerability codes present

${ }^{9}$ Mental health problems excluding major depression

heta adjusted for all other variables presented in the table and nested by GPs' unique identifier 
Age was also associated with a significant increase in wait time ( $\beta$ 1.30, CI 1.10-1.50). Wait time varied according to local health network, for instance patients from LHN A waited 266 days more than those from LHN E $(p=0.0089)$. With regards to morbidity variables, non-vulnerable patients (i.e. with no health conditions) waited 74 more days than medically vulnerable patients before being attached to a GP. Wait times decreased concurrently with increasing urgency of clinical priority level, with Priority 1 patients (most urgent) waiting 442 days less compared to Priority 5 (least urgent) patients. Also, for every additional health condition, patients waited 12 days less for attachment. Wait times were significantly longer for patients with a mental health problem ( $\beta$ 78.50, CI 63.00-94.01), addiction ( $\beta$ 122.07, CI 84.46-159.69) or intellectual disability ( $\beta$ 70.85, CI 26.88-114.82).

\section{Discussion}

In sum, our results show that social and material deprivation are significantly associated with likelihood of attachment to a GP through CWLs and that material deprivation is significantly associated with wait time among patients attached to a GP though CWLs, independently of patients health needs and other control variables. It therefore seems that while CWLs may help reduce inequities in likelihood of attachment to a GP with regards to material deprivation, other inequities remain - our results highlighting a pro-socially advantaged gradient in likelihood of attachment to a GP and a pro-rich gradient in wait time for attachment to a GP. Our findings therefore suggest that CWLs may reduce some inequities, but that, despite a more standardized process for finding a GP, significant socio-economic inequities persist in attachment to a GP through CWLs. While our results do not provide information on the mechanisms leading to these inequities, we can hypothesize that these might be linked to patients' abilities to access health care services, in this case the CWL and GP, as well as to the health care service's characteristics, for instance the CWLs' processes [60].

\section{CWLs may not be reaching patients from materially disadvantaged areas}

The descriptive statistics of our population suggest that, compared to the general population of Quebec (expected $20 \%$ in each deprivation quintile), patients from very materially disadvantaged areas seem underrepresented on the CWLs, while patients from very socially disadvantaged areas seem to be overrepresented. Patients from more materially disadvantaged areas may have lower levels of health literacy [61-67]. This may make it more difficult for them to navigate the complex processes of the health care system $[12,68]$, such as registering on the CWL (e.g. knowing the CWL exists, finding local CWL contact information, filling out form). Moreover, the distribution of this study's patients across deprivation indexes in the five LHNs selected might be different than the distribution observed at the provincial level (20\% in each quintile). A previous report showed that two of the five LHNs were considered socially disadvantaged or socially and materially disadvantaged [69]. The other three LHNs were categorized as being socially and materially advantaged [69]. In order to account for this diversity, we controlled our analysis for the LHN. This may partially explain our finding that patients from more materially deprived areas seem underrepresented on CWLs for attachment to a GP, despite patients with lower income and education levels being more likely to not have a regular GP in Canada [6, 8-11].

Since the study, registration has been centralized provincially (single website) [70], simplifying the process somewhat for patients. However, it has been suggested that health systems should implement measures to better reach patients with low health literacy, not only by simplifying processes, but also by training health providers to better communicate with these patients and disseminating health information through community organisations, schools and the mass-media [71]. Therefore, promoting how to register on the CWL for attachment to a GP through various channels may help reach patients from materially disadvantaged areas.

\section{Patients from materially more advantaged areas and socially more disadvantaged areas less likely to be attached to a GP through CWLs}

Our results also suggest that CWL patients from very materially advantaged and advantaged areas (richest) were significantly less likely to be attached to a GP through the CWLs compared to those from very materially disadvantaged areas (poorest) (OR $0.75, p<0.0001$ and OR 0.88, $p=0.0004$ ), when controlling for health needs, age, sex, local health network and wait time. Conversely, we found that patients from socially very advantaged, advantaged and medium areas were more likely to be attached to a GP compared to patients from very disadvantaged areas (most isolated) (OR 1.23, 1.21 and 1.19 respectively, $p<$ 0.0001). These results are opposite from the results presented in Table 2, suggesting that the control variables have an important influence on the association between social deprivation and likelihood of attachment to a GP, which is expected considering that medical vulnerability and clinical priorities, based on self-reported health conditions are used to assess patient health needs and urgency for attachment and financial incentives are based on patient health status. It is possible that patients from socially disadvantaged areas (more isolated) may be more likely to miss their first appointment with their new GP, which 
officialises the attachment, and are then sent back to the CWL. A recent report on CWL for attachment to a GP in Quebec found in $8.5 \%$ of cases where patients were not attached to the GP to whom they had been assigned, it was because they had missed their appointment [32]. Several studies have found that, in certain populations, patients with lower levels of social support were more likely to miss appointments [72-74]. Also, patients from more socially advantaged areas, by having someone such as a spouse to help explain their health status and advocate for their needs to the CWL nurse, may be more likely to be attached to a GP. However, the literature on the link between social deprivation and access to a GP is virtually inexistent. Our study contributes to this body of knowledge, but the mechanisms by which inequities would occur in likelihood, but not for wait time for attachment to a GP through CWLs remain unclear. Further research is needed to explain these results.

\section{Pro-materially advantaged gradient in wait time for attachment through CWLs}

In terms of wait times for patients who had been attached to a GP through the CWLs, our findings show a significant pro-rich gradient in wait times for attachment, with patients from very materially advantaged areas (richest) having waited an average 34 days less than patients from very disadvantaged areas (poorest) $(p<0.0001)$. For social deprivation, our results show no significant differences other that patients from medium areas waited 15 days less for attachment than those from very disadvantaged areas $(p=0.0061)$. Patients are prioritized by the CWL nurse based on self-reported health information from their registration form and phone evaluation and then selected by the nurse for attachment as GPs become available. Therefore, it may be patients, as previously mentioned, that patients with higher health literacy and communication skills [63-66, 68], often those with higher education, income and from more materially advantaged areas $[61,62]$, are able to report their medical conditions and advocate for their need to be attached more clearly to the nurse which might explain shorter waiting times for attachment. These findings of a pro-rich gradient in wait times aligns with recent literature review by Siciliani which found a few studies that show pro-rich socio-economic inequities in wait times for access for other healthcare services such as elective procedures in publically funded health systems, despite efforts to equalize wait times [75].

\section{Different influences of deprivation on likelihood and wait times}

Moreover, our results highlight that patients from materially disadvantaged areas were more likely to have been attached, but among those who had been attached, they had waited longer. One possible explanation for these seemingly contradictory results is that patients from areas with higher incomes, education levels and employment rates may have stronger abilities to advocate for themselves [63-66]. For instance, they may continue to look for a GP on their own (e.g. asking GPs they see at a walk-in clinic, having family members ask their GPs if they are attaching new patients) and find attachment outside the CWL, in which case the attachment would not appear in the SIGACO database. They would therefore appear to be less likely to be attached to a GP through the CWL when looking at both patients who are still waiting for attachment and those who have been attached. In addition, these seemingly contradictory results in likelihood and wait time may be due to significant differences between patients attached to a GP through CWLs (included in the analysis on wait time) and patients who remained on the CWLs (included in the analysis on likelihood, but not wait time), as suggested by the results in Table 1, which could have influenced the direction of the associations. Another variable that may influence likelihood of attachment is geographic distribution of GPs. However, very little research has been done to analyze the association between area deprivation and supply of GPs. Future research on this topic is warranted.

\section{Inequities in likelihood and wait time for attachment through CWLs for patients with mental health problems, addiction and intellectual disability}

While the focus of our analysis was deprivation, our results also highlight that patients with mental health problems, major depression, addiction and intellectual disability were significantly less likely to be attached to a GP (respectively OR 0.42, $p<0.0001$; OR $0.85, p=0.03$; OR $0.39, p<0.0001$; OR $0.72, p=0.03$ ) and patients with mental health problems, addiction and intellectual disability waited significantly longer for attachment to a GP (respectively 78.50 days, $p<0.0001 ; 122.07$ days, $p<$ $0.0001 ; 70.85$ days, $p=0.0019)$ compared to patients without these conditions, when controlling for deprivation, clinical priority level and other variables. This suggests that these patients with complex psychosocial needs face certain inequities in attachment to a GP through CWLs. It seems essential that qualitative research be done to better understand the barriers these patients face, particularly as some of these conditions seem more prevalent in patients from high deprivation areas (Table 2).

Including socio-economic status as a prioritization criteria Our findings suggest that CWLs have not been entirely successful at reducing socio-economic inequities by prioritizing attachment to a GP based on patients' medical 
needs, as we found significant differences in likelihood of being attached between quintiles of both social and material deprivation and wait time for attachment was significantly influenced by material deprivation. In a study by Noseworthy et al., it was reported that stakeholders (public and health administrators) felt that socio-economic status should not be used to prioritize patients for access to healthcare services because, if the guidelines were applied rigorously, patients' needs would adequately be captured by medical criteria [22]. Therefore, one avenue to reduce inequities in attachment to a GP through CWLs may be to improve the respect of prioritization guidelines.

In Aidem's study, while some stakeholders (public, practitioners, administrators, policy-makers) felt that while socio-economic status should not be included as a criterion, most agreed that conditions that disproportionately affect socio-economically disadvantaged groups such as mental health problems and addiction should be prioritized [76]. While this may be a solution to consider for policy, our results show that patients with mental health problems and addiction are less likely to be attached to a GP and wait longer for attachment even when controlling for area material and social deprivation, and therefore prioritizing these conditions in CWLs may not be sufficient to reduce inequities in attachment to a GP.

In Quebec, recent changes in provincial policies of family medicine groups now weigh patients from high social and material deprivation (i.e. poorest, most isolated) areas more heavily for resource allocations. Therefore, including socio-economic factors as criteria to prioritize patients in CWLs, albeit with less weight than medical criteria, may be acceptable to stakeholders and may be a potential solution to improving equity in attachment to a GP. An evaluation of this change in policy should be conducted to examine whether it acts as an incentive for GPs to attach patients from more disadvantaged areas.

\section{Study strengths and limitations}

Our analysis has several limitations. First, the deprivation index is based on census data at the neighbourhood level and is used as a proxy measure for socio-economic status of individual patients $[11,41,49,50]$. It is therefore possible for patients to live in a materially or socially disadvantaged area, but to have a higher socio-economic status or to have strong social support. In addition, as reported by Pampalon et al., in less population areas, social health inequalities could be underestimated using this index $[41,49]$. In our study, one local health network (D) was located in a semi-urban area which may have affected our results and led to an underestimation of deprivation. However, by controlling for local health network, we minimized this potential bias. Furthermore, Pampalon's social and material deprivation indexes do not include other patient characteristics such as immigration status or ethnicity which have been found to influence access to PHC in other studies [77-83]. While there are limitations to using the deprivation index, socio-economic variables (e.g. income, education, employment status, marital status) are often unavailable in administrative databases used by policy-makers. Second, our analysis focused on attachment to a GP through CWLs. However, formal attachment to a GP does not guarantee access to this GP and may therefore not fully reflect access to PHC. Continued access to the GP after attachment was not captured in the administrative database used, but warrants further research. Future research should evaluate the impact of attachment through these CWLs using longitudinal data. In addition, the administrative database used does not provide information on attachment to a GP through means other than the CWL (e.g. by asking a GP at a walk-in clinic). Patients registered on the CWLs may have found attachment to a GP elsewhere, but remain on the waiting list. Finally, many factors may influence attachment to a GP through CWLs such as geographic distribution and availability of primary care workforce and prioritization processes, etc. These factors, not captured by our variables, may interact with social and material deprivation and influence likelihood and wait time for attachment to a GP.

\section{Conclusions}

Our findings suggest that centralized waiting lists have not been successful at standardizing the process of attachment to a GP sufficiently to eliminate socio-economic inequities. Policy makers should take these findings into consideration to adjust CWL processes. Further research on the potential mechanisms explaining these inequities, such as availability of GPs in disadvantaged areas, physicians' willingness to attach disadvantaged patients and barriers disadvantaged patients may face in using CWLs is necessary to inform the implementation of potential solutions.

\begin{abstract}
Abbreviations
Cl: Confidence interval; COPD: Chronic obstructive pulmonary disease; CWL: Centralized waiting list; GP: General Practitioner; LHN: Local health network; OECD: Organization for Economic Co-Operation and Development; OR: Odds ratio; PHC: Primary health care; RAMQ: Régie de l'assurance maladie du Québec (Quebec's Health Insurance Board); S.D.: Standard deviation; SIGACO: Système d'information des guichets d'accès pour la clientele orpheline (Quebec's information system for centralized waiting lists for unattached patients)
\end{abstract}

\section{Acknowledgements}

We wish to thank the Quebec Population Health Research Network for its financial support in publishing this article. We would like to acknowledge the engagement of all the decision-makers involved in this study. We would also like to thank our colleagues Danièle Roberge, Élizabeth Côté-Boileau and Catherine Lamoureux-Lamarche who were of great help at different stages of this study. 


\section{Funding}

This study was funded by the Fonds de recherche du Québec-Santé (FRQ-S Grant \#28974) for which MB is the principal investigator. MAS received a doctoral award from the FRQ-S and from Quebec's Support for People and Patient-Oriented Research and Trials Unit. MB holds a Canada Research Chair in Clinical Governance in Primary Health Care. ABoivin holds a Canada Research Chair in Patient and Public Partnership. The publication of this article was funded by the Quebec Population Health Research Network.

\section{Availability of data and materials}

The data that support the findings of this study are not publicly available due to them containing individual-level information that could compromise patients' privacy.

\section{Authors' contributions}

MAS conceptualized the analyses, interpreted the results and drafted the manuscript. MB conceived the larger study on CWLs and all authors participated in its design. MB, ABrousselle, ABoivin, CAD, CL, KN and NT commented on and helped revise the article. DB conducted the statistical analyses in collaboration with MAS. All authors read and approved the final manuscript.

\section{Ethics approval and consent to participate}

This study was approved by the Research Ethics Committee of Sherbrooke University Hospital Centre (ref. number MP-31-2015-819: 14-091), Permission to access to the SIGACO database used in this study was granted by the five LHNs included in the study and was approved by the Research Ethics Committee of Sherbrooke University Hospital Centre.

\section{Consent for publication}

\section{Not applicable.}

\section{Competing interests}

The authors declare that they have no competing interests.

\section{Publisher's Note}

Springer Nature remains neutral with regard to jurisdictional claims in published maps and institutional affiliations.

\begin{abstract}
Author details
${ }^{1}$ Centre de recherche Charles-Le Moyne - Saguenay Lac-St-Jean sur les innovations en santé, Université de Sherbrooke, Longueuil Campus, 150 Place Charles-Le Moyne, Suite 200, Longueuil, Quebec J4K 0A8, Canada. ${ }^{2}$ School of Public Administration, University of Victoria, 3800 Finnerty Rd, Suite A302, Victoria, British Columbia V8P 5C2, Canada. ${ }^{3}$ Centre de recherche sur la gouvernance, École nationale d'administration publique, 4750, Avenue Henri-Julien, Office 5117, Montreal, Quebec H2T 3E5, Canada. ${ }^{4}$ Centre de recherche du Centre Hospitalier de I'Université de Montréal, Université de Montréal, 900 Rue Saint-Denis, Montreal, Quebec H2X 0A9, Canada.

${ }^{5}$ Direction de santé publique, Centre intégré de santé et des services sociaux - Montérégie-Centre, 1255 rue Beauregard, Longueuil, Quebec J4K 2M3, Canada. ${ }^{6}$ Faculty of Nursing, Université de Montréal, 2375, chemin de la Côte Ste-Catherine, Office 5103, Montreal, Quebec H3T 1A8, Canada.
\end{abstract}

\section{Received: 2 April 2018 Accepted: 5 November 2018} Published online: 04 December 2018

\section{References}

1. Sheiham A. Closing the gap in a generation: health equity through action on the social determinants of health. A report of the WHO Commission on social determinants of health (CSDH) 2008. Community Dent Health. 2009;26:2-3.

2. van Doorslaer E, Masseria C, Koolman X. For the OHERG: inequalities in access to medical care by income in developed countries. CMAJ : Canadian Medical Association Journal. 2006;174:177-83.

3. Asada Y, Kephart G. Equity in health services use and intensity of use in Canada. BMC Health Serv Res. 2007:7:41.

4. Birch S, Eyles J, Newbold KB. Equitable access to health care: methodological extensions to the analysis of physician utilization in Canada. Health Econ. 1993;2:87-101.

5. Allin S. Does equity in healthcare use vary across Canadian provinces? Healthcare Policy. 2008:3:83-99.
6. Dunlop S, Coyte PC, Mclsaac W. Socio-economic status and the utilisation of physicians' services: results from the Canadian National Population Health Survey. Soc Sci Med. 2000;51:123-33.

7. Lambrey JM, Defriese GH, Carey TS, Rlicketts TC, Biddle AK. The effects of having a regular doctor on access to primary care. Med Care. 1996;34:138-51.

8. Talbot Y, Fuller-Thomson E, Tudiver F, Habib Y, Mclsaac WJ. Candians without regular medical doctors - who are they? Can Fam Physician. 2001;47:58-64.

9. Hay C, Pacey M, Bains N. Understanding the unattached population in Ontario: evidence from the primary care access survey (PCAS). HealthCare Policy. 2010;6:33-47.

10. Dubé-Linteau A, Pineault R, Levesque J-F, Lecours C, Tremblay M-E. Enquête québécoise Sur l'expérience de soins 2010-2011. Le médecin de famille et l'endroit habituel de soins : regard Sur l'expérience vécue par les Québécois. Vol. 2. Pp. 73. Québec: Institut de la statistique du Québec; 2013. p. 73.

11. Pampalon R, Raymond G. A deprivation index for health and welfare planning in Quebec. Chronic Diseases and Injuries in Canada. 2000;21:104.

12. Ouimet M-J, Pineault R, Prud'homme A, Provost S, Fournier M, Levesque J-F. The impact of primary healthcare reform on equity of utilization of services in the province of Quebec: a 2003-2010 follow-up. Int J Equity Health. 2015;14:139.

13. Breton M, Green M, Kreindler S, Sutherland J, Jbilou J, Wong ST, Shaw J, Crooks VA, Contandriopoulos D, Smithman MA, Brousselle A. A comparative analysis of centralized waiting lists for patients without a primary care provider implemented in six Canadian provinces: study protocol. BMC Health Serv Res. 2017;17:60.

14. Access to a regular medical doctor - Canadian Community Health Survey 2013-2014 [http://www.statcan.gc.ca/pub/82-625-x/2014001/article/14013eng.htm], Accessed on February 2, 2018.

15. Commonwealth Fund International Health Policy Survey, Schoen C, et al. Toward higher-performance health systems: adults' health care experiences in seven countries, 2007. Health Aff. 2007;26:W717-34.

16. Régie de l'assurance maladie du Québec: Rapport 6 - Statut des inscriptions auprès d'un médecin de famille. 2014.

17. Roy $\mathrm{A}$, Breton M, Loslier J. Providing continuity of care to a specific population: attracting new family physicians. Can Fam Physician. 2016;62:e256-62.

18. Collège des médecins de famille du C. Conseil pratique sur l'inscription de clientèle en médecine familiale. vol. In: 2013; 2012.

19. Régie de l'assurance maladie du Québec: Rapport 1 - Statut des inscriptions auprès d'un médecin de famille en date du 2015-03-31. 2015.

20. Breton M, Wong ST, Smithman MA, Kreindler S, Jbilou J, Marshall EG, Sutherland J, Brousselle A, Shaw J, Crooks VA, et al. Centralized waiting lists for unattached patients in primary care: learning from an intervention implemented in seven Canadian provinces. Healthc Policy. 2018;13:65-82.

21. Dew K, Cumming J, McLeod D, Morgan S, McKinlay E, Dowell A, Love T. Explicit rationing of elective services: implementing the New Zealand reforms. Health Policy. 2005;74:1-12.

22. Noseworthy TW, McGurran JJ, Hadorn DC. Waiting for scheduled services in Canada: development of priority-setting scoring systems. J Eval Clin Pract. 2003;9:23-31.

23. Novak K, Veldhuyzen Van Zanten S, Pendharkar SR. Improving access in gastroenterology: the single point of entry model for referrals. Can J Gastroenterol. 2013;27:633-5.

24. Raymond $M-H$, Feldman D, Prud'homme M-P, Demers L, Hunt LA, Bissett M, Slater CE. Who's next? Referral prioritisation criteria for home care occupational therapy. Int J Ther Rehabil. 2013;20:580-8.

25. Oniscu GC, Schalkwijk AAH, Johnson RJ, Brown H, Forsythe JLR. Equity of access to renal transplant waiting list and renal transplantation in Scotland: cohort study. BMJ. 2003;327:1261.

26. Arnett G, Hadorn DC. Developing priority criteria for hip and knee replacement: results from the Western Canada waiting list project. Can J Surg. 2003:46:290-7.

27. Askildsen JE, Holmås TH, Kaarboe O: Prioritization and patients' rights: analysing the effect of a reform in the Norwegian hospital sector. Soc Sci Med 2010, 70:199-208 110p.

28. Januleviciute J, Askildsen JE, Kaarboe O, Holmås TH, Sutton M: The impact of different prioritisation policies on waiting times: case studies of Norway and Scotland. Soc Sci Med 2013, 97:1-6 6p.

29. Breton M, Brousselle A, Boivin A, Loignon C, Touati N, Dubois C-A, Nour K, Berbiche D, Roberge D. Evaluation of the implementation of centralized waiting lists for patients without a family physician and their effects across the province of Quebec. Implement Sci. 2014;9:117. 
30. Breton M, Smithman MA, Brousselle A, Loignon C, Touati N, Dubois C-A, Nour K, Boivin A, Berbiche D, Roberge D. Assessing the performance of centralized waiting lists for patients without a regular family physician using clinical-administrative data. BMC Fam Pract. 2017;18:1.

31. 1 million de personne de plus ont maintenant accès à un médecin de famille [http://msss.gouv.qc.ca/documentation/salle-de-presse/ ficheCommunique.php?id=1461], Accessed on March 27, 2018.

32. Près de 75000 patients refusés par les médecins de famille [http://ici.radiocanada.ca/nouvelle/1084272/patients-refuses-guichet-acces-medecin-famillequebec], Accessed on March 22, 2018.

33. Régie de l'assurance maladie du Québec. Catégories de problèmes de santé. Entente particulière relative aux services de médecine de famille, de prise en charge et de suivi de la clientèle. In: Québec: RAMQ; 2012.

34. Régie de l'assurance maladie du Québec: Lettre d'entente no 245 Concernant la prise en charge et le suivi de tout patient sans médecin de famille Sur référence ou non du guichet d'accès du CSSS; 2013.

35. Breton M, Brousselle A, Boivin A, Roberge D, Pineault R, Berbiche D. Who gets a family physician through centralized waiting lists? BMC Fam Pract. 2015;16.

36. Gouvernement du Québec. Report of the auditor general of Québec to the National Assembly for 2015-2016. Chapter. 2016:5.

37. Canada's Health Care System [http://www.hc-sc.gc.ca/hcs-sss/pubs/systemregime/2011-hcs-sss/index-eng.php.], Accessed on January 20, 2018.

38. Statistics Canada: Population by year, by province and territory. CANSIM ted.; 2017.

39. Breton M, Maillet L, Haggerty J, Vedel I. Mandated local health networks across the province of Québec: a better collaboration with primary care working in the communities? London Journal of Primary Care. 2014;6:71-8.

40. Kawachi I, Berkman LF. Neighborhoods and health: Oxford University Press; 2003.

41. Pampalon R, Hamel D, Gamache P, Raymond G. A deprivation index for health planning in Canada. Chronic Dis Can. 2009;29:178-91.

42. Krieger N, Chen JT, Waterman PD, Soobader M-J, Subramanian S, Carson R. Geocoding and monitoring of US socioeconomic inequalities in mortality and cancer incidence: does the choice of area-based measure and geographic level matter? The public health disparities geocoding project. Am J Epidemiol. 2002;156:471-82.

43. Krieger N, Williams DR, Moss NE. Measuring social class in US public health research: concepts, methodologies and guidelines. Annu Rev Public Health. 1997;18:341-78.

44. Mustard CA, Derksen S, Berthelot J-M, Wolfson M. Assessing ecologic proxies for household income: a comparison of household and neighbourhood level income measures in the study of population health status. Health \& place. 1999:5:157-71.

45. Messer LC, Laraia BA, Kaufman JS, Eyster J, Holzman C, Culhane J, Elo I, Burke JG, O'campo P. The development of a standardized neighborhood deprivation index. J Urban Health. 2006;83:1041-62.

46. Havard S, Deguen S, Bodin J, Louis K, Laurent O, Bard D. A small-area index of socioeconomic deprivation to capture health inequalities in France. Soc Sci Med. 2008:67:2007-16.

47. Salmond CE, Crampton P. Development of New Zealand's deprivation index (NZDep) and its uptake as a national policy tool. Canadian Journal of Public Health/Revue Canadienne de Sante'e Publique. 2012:S7-S11.

48. Cubbin C, Hadden WC, Winkleby MA. Neighborhood context and cardiovascular disease risk factors: the contribution of material deprivation. Ethnicity \& disease. 2001;11:687-700.

49. Pampalon R, Hamel D, Gamache P, Philibert MD, Raymond G, Simpson A. An area-based material and social deprivation index for public health in Québec and Canada. Canadian Journal of Public Health/Revue Canadienne de Sante'e Publique. 2012:S17-22.

50. Pampalon R, Hamel D, Gamachem P. The Québec index of material and social deprivation. Methodological Follow-up, 1991 Through. 2011:2006.

51. Townsend P. Deprivation. J Soc Policy. 1987;16:125-46.

52. Pampalon R, Hamel D, Gamache P. A comparison of individual and areabased socio-economic data for monitoring social inequalities in health. Statistics Canada Health Reports. 2009;20:85-94.

53. Pampalon R, Raymond G. Un indice de défavorisation pour la planification de la santé et du bien-être au Québec. Maladies chroniques au Canada. 2000;21:104-13.

54. Information $\mathrm{ClfH}$. Reducing gaps in health: a focus on socio-economic status in urban Canada. In: Ottawa: $\mathrm{ClHI} ; 2008$.
55. Predy G, Edwards J, Fraser-Lee N, Ladd B, Moore K, Lightfoot P, Spinola C. Poverty and health in Edmonton. Edmonton: Publiuc Health Division Alberta Health Services; 2008.

56. Pampalon R, Raymond $\mathrm{G}$. Indice de défavorisation matérielle et sociale: son application au secteur de la santé et du bien-être. Santé, société et solidarité. 2003;2:191-208.

57. Philibert MD, Pampalon R, Hamel D, Thouez JP, Loiselle CG. Material and social deprivation and health and social services utilisation in Québec: a local-scale evaluation system. Soc Sci Med. 2007;64:1651-64.

58. Gamache P, Hamel D: The challenges of updating the deprivation index with data from the 2011 census and the National Household Survey (NHS). Bureau d'information et d'études en santé des populations; 2017.

59. Heck RH, Thomas SL, Tabata LN. Multilevel and Longitudinal Modeling with IBM SPSS. Taylor \& Francis; 2013.

60. Levesque J-F, Harris MF, Russell G. Patient-centred access to health care: conceptualising access at the interface of health systems and populations. Int J Equity Health. 2013;12:18-26.

61. Protheroe J, Whittle R, Bartlam B, Estacio EV, Clark L, Kurth J. Health literacy, associated lifestyle and demographic factors in adult population of an English city: a cross-sectional survey. Health Expectations : An International Journal of Public Participation in Health Care and Health Policy. 2017;20:112-9.

62. Rowlands G, Protheroe J, Winkley J, Richardson M, Seed PT, Rudd R. A mismatch between population health literacy and the complexity of health information: an observational study. Br J Gen Pract. 2015;65:e379-86.

63. Champlin S, Mackert M, Glowacki EM, Donovan EE. Toward a better understanding of patient health literacy a focus on the skills patients need to find health information. Qual Health Res. 2017;27:1160-76.

64. Eichler K, Wieser S, Brügger U. The costs of limited health literacy: a systematic review. International journal of public health. 2009;54:313-24.

65. Fetter MS. Promoting health literacy with vulnerable behavioral health clients. Issues in mental health nursing. 2009;30:798-802.

66. Levy H, Janke A. Health literacy and access to care. J Health Commun. 2016; 21:43-50.

67. World Health Organization. Health literacy toolkit for low- and middleincome countries. A series of information sheets to empower communities and strengthen health systems. Geneva: World Health Organization; 2015

68. Loignon C, Hudon C, Goulet E, Boyer S, Laat M, Fournier N. Perceived barriers to healthcare for persons living in poverty in Quebec, Canada: the EQUlhealThy project. Int J Eq Health. 2015;14.

69. Roberge D, Pineault R, Hamel M, Borgès Da Silva R, Cazale L, Levesque J-F, Ouellet $D$. L'accessibilité et la continuité des services de santé: Une étude Sur la première ligne au Québec. Rapport méthodologique de l'analyse des contextes. Montréal: Centre de recherche de l'Hôpital Charles LeMoyne, Agence de la santé et des services sociaux de Montréal-Direction de santé publique, Institut national de santé publique du Québec; 2007.

70. Ministère de la santé et des services sociaux. Inscription auprès d'un médecin de famille. In: Gouvernement du Québec; 2016.

71. Paasche-Orlow MK, Schillinger D, Greene SM, Wagner EH. How health care systems can begin to address the challenge of limited literacy. J Gen Intern Med. 2006;21:884-7.

72. Waller D, Agass M, Mant D, Coulter A, Fuller A, Jones L. Health checks in general practice: another example of inverse care? Bmj. 1990;300:1115-8.

73. Odonkor CA, Christiansen S, Chen Y, Sathiyakumar A, Chaudhry H, Cinquegrana D, Lange J, He C, Cohen SP. Factors associated with missed appointments at an academic pain treatment center: a prospective yearlong longitudinal study. Anesth Analg. 2017;125:562-70.

74. Ford JA, Turley R, Porter T, Shakespeare T, Wong G, Jones AP, Steel N. Access to primary care for socio-economically disadvantaged older people in rural areas: a qualitative study. PLoS One. 2018;13:e0193952.

75. Siciliani L. Waiting times: evidence of social inequalities in access for care. In: Sobolev B, Levy A, Goring S, editors. Data and measures in health services research. Boston, MA: Springer US; 2016. p. 1-17.

76. Aidem JM. Stakeholder views on criteria and processes for priority setting in Norway: a qualitative study. Health Policy. 2017;121:683-90

77. Mian O, Pong R. Does better access to FPs decrease the likelihood of emergency department use?: results from the primary care access survey. Can Fam Physician. 2012;58:e658-66.

78. Tiagi R. Access to and utilization of health care services among Canada's immigrants. International Journal of Migration, Health and Social Care. 2016;12:146-56. 
79. Baker R, Bankart M, Rashid A, Banerjee J, Conroy S, Habiba M, Hsu R, Wilson A, Agarwal S, Camosso-Stefinovic J. Characteristics of general practices associated with emergency-department attendance rates: a cross-sectional study. BMJ Qual Saf. 2011:20:953-8.

80. Jatrana S, Crampton P. Affiliation with a primary care provider in New Zealand: who is, who isn't. Health Policy. 2009;91:286-96.

81. Jatrana S, Crampton P, Richardson K. Continuity of care with general practitioners in New Zealand: results from SoFIE-primary care. The New Zealand Medical Journal. 2011;124.

82. Ruud SE, Hjortdahl P, Natvig B. Reasons for attending a general emergency outpatient clinic versus a regular general practitioner-a survey among immigrant and native walk-in patients in OsloNorway. Scand J Prim Health Care. 2017;35:35-45.

83. Talbot Y, Fuller-Thomson E, Tudiver F, Habib Y, Mclsaac WJ. Canadians without regular medical doctors. Who are they? Can Fam Physician. 2001;47:58-64.

Ready to submit your research? Choose BMC and benefit from:

- fast, convenient online submission

- thorough peer review by experienced researchers in your field

- rapid publication on acceptance

- support for research data, including large and complex data types

- gold Open Access which fosters wider collaboration and increased citations

- maximum visibility for your research: over $100 \mathrm{M}$ website views per year

At BMC, research is always in progress.

Learn more biomedcentral.com/submissions 